\title{
Improvement of Immunomagnetic Separation for Escherichia coli 0157:H7 Detection by the PickPen Magnetic Particle Separation Device ${ }^{\dagger}$
}

\author{
XIANGWU NOU, $\ddagger$ TERRANCE M. ARTHUR,* JOSEPH M. BOSILEVAC, DAYNA M. BRICHTA-HARHAY, \\ MICHAEL N. GUERINI, NORASAK KALCHAYANAND, AND MOHAMMAD KOOHMARAIE
}

U.S. Department of Agriculture, Agricultural Research Service, Roman L. Hruska U.S. Meat Animal Research Center, Spur 18-D, P.O. Box 166, Clay Center, Nebraska 68933-0166, USA

MS 06-057: Received 31 January 2006/Accepted 26 April 2006

\begin{abstract}
Conventional immunomagnetic separation (IMS) procedures, which use an external magnetic source to capture magnetic particles against the side of a test tube, are labor-intensive and can have poor sensitivity for the target organism because of high background microflora that is not effectively washed away during the IMS process. This report compares the conventional IMS procedure to a new IMS procedure with an intrasolution magnetic particle transfer device, the PickPen. The IMS target for the majority of these studies is Escherichia coli O157:H7 in various types of samples, including cattle feces, hides, carcasses, and ground beef. Comparison of the two IMS methods showed a significant difference $(P<0.05)$ in the efficiency of detecting E. coli $\mathrm{O} 157: \mathrm{H} 7$ from cattle carcass surface, cattle hide, and cattle fecal samples. No significant improvement $(P$ $>0.05)$ in E. coli $\mathrm{O} 157: \mathrm{H} 7$ detection was observed when the PickPen IMS procedure was used to isolate this pathogen from ground beef samples. Use of the PickPen IMS greatly increases the throughput of the IMS procedure and may be more compatible with various emerging technologies for pathogen detection. In addition, the efficacy of sequential IMS for multiple pathogens is reported herein.
\end{abstract}

Shiga toxin-producing Escherichia coli $\mathrm{O} 157: \mathrm{H} 7$ is a significant foodborne pathogen that has emerged in the past two decades. E. coli O157:H7 infections have been linked to the consumption of undercooked meat and dairy products, contaminated fresh produce and fruit juices, as well as to exposure to contaminated farm and zoo animals and recreational water $(1,9,11,14)$. Cattle are generally considered the primary reservoir of $E$. coli $\mathrm{O} 157: \mathrm{H} 7$. In several recent surveys, researchers found that over $70 \%$ of cattle hides may be contaminated with $E$. coli $\mathrm{O} 157: \mathrm{H} 7$ at the beginning of the slaughter process $(2,4)$.

The implementation of hazard analysis critical control point (HACCP) programs in meat-processing facilities has played a major role in decreasing the incidence of $E$. coli O157:H7 contamination of retail beef products and human sickness (12). Process control and end product testing are integral parts of the HACCP programs in beef cattle processing establishments. Rapid and sensitive detection of $E$. coli $\mathrm{O} 157: \mathrm{H} 7$ at various stages of processing and from a variety of meat products is a critical requirement for ensuring the safety of beef products.

Immunomagnetic separation (IMS) has significantly

\footnotetext{
* Author for correspondence. Tel: 402-762-4227; Fax: 402-762-4149; E-mail: arthur@email.marc.usda.gov.

$\dagger$ Mention of trade names or commercial products in this article is solely for the purpose of providing specific information and does not imply recommendation or endorsement by the U.S. Department of Agriculture.

$\$$ Present address: U.S. Department of Agriculture, Agricultural Research Service, Animal \& Natural Resource Institute, Building 201, BARCEast, 10300 Baltimore Avenue, Beltsville, MD 20705-2350, USA.
}

improved the sensitivity of $E$. coli $\mathrm{O} 157: \mathrm{H} 7$ detection (7, 8). Traditionally, the IMS procedure consisted of mixing a sample volume with antibody-coated paramagnetic beads in a microcentrifuge tube. Following capture of the target by the beads, a magnet was applied to the side of the tube to hold the magnetic beads in place while the liquid sample was removed from the tube. This procedure was repeated to wash the beads. Following a series of wash steps, the beads were resuspended in a small volume (approximately $100 \mu \mathrm{l})$ of buffer and then plated onto selective or differential agar media for the detection of the bacteria of interest. While this procedure is not technically complicated, traditional IMS was a labor-intensive process and not amenable to high sample throughput. Many modifications of the standard IMS procedure have been made, including modifications in apparatus, bead sizes, and washing procedures $(3,6,10)$. BioNobile (Turku, Finland) has recently developed an intrasolution magnetic particle separation device, called the PickPen, which has primarily been used for rapidly transferring DNA, RNA, and protein molecules linked to magnetic particles. BioControl Systems (Bellevue, Wash.) adopted this intrasolution magnetic particle separation device in their commercial E. coli O157:H7 Assurance GDS products. In this study, we show that the incorporation of the PickPen in the IMS process can improve the procedure by increasing sample throughput and decreasing carryover of background microflora without compromising the sensitivity. We also demonstrate an extended application of this procedure, involving sequential IMS that allows the rapid isolation of multiple target pathogens from a single sample. 


\section{MATERIALS AND METHODS}

Bacterial strains. E. coli $0157: \mathrm{H} 7$ strains from the U.S. Meat Animal Research Center's Meats Research Unit strain collection were used to spike some samples as indicated in the text. The strains used in these studies (114AC1 and 131AC1) were isolated from beef carcasses during processing at commercial slaughter plants. The strains were grown separately overnight and then combined prior to spiking the samples.

Samples. Ground beef ( $85 \%$ lean) was purchased from local retailers 24 to $72 \mathrm{~h}$ prior to the initiation of each experiment. Preevisceration carcass surface sponge samples were obtained from large commercial beef processing plants. The carcass surface sponge samples included samples taken both prior to and after hot water treatments, lactic acid treatments, or both following hide removal. Hide sponge samples and fecal samples were collected from feedlot cattle restrained in a squeeze chute. Hides, feces, and carcasses were sampled as previously described (4).

Ground beef samples and preevisceration carcass sponge samples were enriched to simulate the normal background flora that would be present in these samples. An overnight culture of E. coli $\mathrm{O} 157: \mathrm{H} 7$ was serially diluted and mixed with $5 \mathrm{ml}$ of the enrichment culture to compare the recovery of varying target cell levels by the two IMS methods. The spiked samples were vortexed, and the inoculated E. coli O157:H7 cells were recovered by either the conventional IMS (C-IMS) or the IMS with the PickPen (P-IMS) procedure. Ground beef enrichments were inoculated with levels from 80 to $40,000 \mathrm{CFU} / \mathrm{ml}$, while preevisceration sample enrichments were inoculated with 32 to $6,400 \mathrm{CFU} / \mathrm{ml}$. After the 5-ml aliquots were inoculated and mixed, $1 \mathrm{ml}$ was removed for each IMS method.

Ground beef samples were also spiked prior to enrichment with various E. coli $\mathrm{O} 157: \mathrm{H} 7$ strains at low levels (1 to $2 \mathrm{CFU} /$ 65 -g sample). Spiked ground beef samples $(65 \mathrm{~g})$ were processed in the following groups: (i) E. coli $\mathrm{O} 157: \mathrm{H} 7$ inoculated at 2.0 CFU/65 $\mathrm{g}$ and then enriched in $185 \mathrm{ml}$ of buffered peptone water (Difco, Becton Dickinson Microbiology Systems, Sparks, Md.) for $4 \mathrm{~h}$ at $37^{\circ} \mathrm{C}$ with shaking, with anti-O157 Dynabeads (Invitrogen, Brown Deer, Wis.) used for IMS; (ii) E. coli O157:H7 inoculated at $1.4 \mathrm{CFU} / 65 \mathrm{~g}$ and then enriched in $185 \mathrm{ml}$ of buffered peptone water for $4 \mathrm{~h}$ at $37^{\circ} \mathrm{C}$ with shaking, with E. coli $\mathrm{O} 157$ A-Beads (Immtech Inc., New Windsor, Md.) used for IMS; and (iii) E. coli O157:H7 inoculated at 1.7 CFU/65 $\mathrm{g}$ and then enriched in 500 $\mathrm{ml}$ of BAX enrichment medium (DuPont Qualicon, Wilmington, Del.) for $24 \mathrm{~h}$ at $37^{\circ} \mathrm{C}$ without aeration, with anti-O157 Dynabeads used for IMS.

Preevisceration carcass sponge samples, hide sponge samples, and fecal samples (10 g) were not inoculated and were enriched in appropriate volumes of tryptic soy broth (Difco, Becton Dickinson) as previously described (6).

IMS. C-IMS was performed, as previously described (5), with enrichment cultures from different sample types. P-IMS was performed as follows: $1 \mathrm{ml}$ from each enrichment was placed in individual wells in a 96-well deep well (2-ml capacity) microtiter plate. The microtiter plate wells were preloaded with $20 \mu \mathrm{l}$ of magnetic beads coated with anti-E. coli O157 antibody (Dynal, Lake Success, N.Y., unless otherwise indicated). The beads were incubated with the culture for $15 \mathrm{~min}$ at room temperature with moderate $(\sim 800 \mathrm{rpm})$ shaking on a microplate shaker. Magnetic beads were captured with an eight-channel PickPen (BioNobile) magnetic particle separation device by gently stirring the culture in an up-and-down corkscrew motion for $30 \mathrm{~s}$. The captured beads were then washed by releasing and recapturing the beads in wells containing $1 \mathrm{ml}$ of washing buffer (phosphate-buffered salineTween 20). The wash step was repeated with new washing wells. The washed beads were released in a microplate well containing $100 \mu \mathrm{l}$ of the washing buffer. Fifty microliters of the beads was plated on sorbitol MacConkey agar (Difco, Becton Dickinson) supplemented with cefixime $(0.05 \mathrm{mg} / \mathrm{liter}$; Dynal $)$ and potassium tellurite (2.5 mg/liter; Dynal) and on CHROMagar (DRG International Inc., Mountainside, N.J.) supplemented with tellurite (1 mg/liter; Sigma, St. Louis, Mo.) and novobiocin (5 mg/liter; Sigma). Characteristic colonies on both plates were identified as $E$. coli $\mathrm{O} 157$ by the Oxoid Dry-Spot O157 assay kit (Oxoid, Basingstoke, UK).

Sequential IMS for target pathogens. To test the ability to perform sequential IMS on a sample, uninoculated enrichments of cattle hide samples were used. Enrichments $(2 \times 1 \mathrm{ml})$ were used, with $1 \mathrm{ml}$ receiving $E$. coli beads first and then Salmonella; the order was reversed for the second aliquot. The beads were extracted from enrichment samples with the PickPen device as described above. Next, $20 \mu \mathrm{l}$ of either Salmonella or E. coli O157:H7 beads (whichever was not previously used) was added to the enrichments, and this new set of beads was recovered with the PickPen. The recovered beads were processed for the detection of either Salmonella or E. coli O157:H7. For E. coli O157:H7, the final bead-bacteria complexes were spread plated onto (i) CHROMagar supplemented with tellurite (1 mg/liter; Sigma) and novobiocin (5 mg/liter) or (ii) sorbitol MacConkey agar supplemented with cefixime $(0.05 \mathrm{mg} / \mathrm{liter})$ and potassium tellurite $(2.5$ $\mathrm{mg} / \mathrm{liter}$ ). For Salmonella, the IMS beads were transferred to Rappaport-Vassiliadis soya (Difco, Becton Dickinson) broth and incubated at $42^{\circ} \mathrm{C}$ overnight. Salmonella present in these samples was detected by swabbing the inoculated Rappaport-VassiliadisSoya broth onto (i) Hektoen Enteric agar (Difco, Becton Dickinson) with novobiocin at $5 \mathrm{mg} / \mathrm{liter}$ and (ii) brilliant green medium with sulfadiazine (Difco, Becton Dickinson).

Statistical analysis. Pairwise comparisons of frequencies of E. coli $\mathrm{O} 157$ detection between the two IMS methods were made by the PROC FREQ and Mantel-Haenszel chi-square analysis procedures of SAS (SAS Institute, Inc., Cary, N.C.).

\section{RESULTS AND DISCUSSION}

The PickPen device has an eight-channel design that is compatible with 96-well microtiter plates. In our experiments, it typically takes less than 2 min to process eight samples after initial incubation of the sample aliquot with the immunobeads. This represents a major improvement in terms of sample throughput for IMS. In most cases, we also observed a more consistent recovery of beads for P-IMS than for C-IMS. In C-IMS procedures, bead recovery is sometimes variable. Because a stationary external magnet is used to recover the beads, there are conditions in which the beads are not sufficiently held in place by the magnet and are lost during aspiration of the enrichment culture or wash buffer. The capture of beads via an intrasolution magnetic device alleviates this problem.

Comparison of C-IMS and P-IMS for the recovery of increasing concentrations of $E$. coli $0157: \mathrm{H7}$ from enriched cultures. Spiked ground beef samples and preevisceration carcass sponge samples were subjected to either the C-IMS or P-IMS procedure for a comparison of $E$. coli $\mathrm{O} 157: \mathrm{H} 7$ recovery. Table 1 shows that the two methods 
TABLE 1. Recovery of E. coli O157:H7 from inoculated enrichments

\begin{tabular}{|c|c|c|c|c|}
\hline \multirow{2}{*}{$\begin{array}{l}\text { Source of } \\
\text { enrichment }\end{array}$} & \multirow{2}{*}{$\begin{array}{l}\text { Inoculum } \\
\text { (CFU/ml) }\end{array}$} & \multirow{2}{*}{$\begin{array}{l}\text { No. of } \\
\text { samples }\end{array}$} & \multicolumn{2}{|c|}{$\begin{array}{c}\text { No. (\%) of E. coli } \mathrm{O} 157: \mathrm{H} 7 \\
\text { recovered }^{a}\end{array}$} \\
\hline & & & C-IMS & P-IMS \\
\hline \multirow[t]{7}{*}{ Ground beef } & 80 & 8 & $6(75)$ A & 7 (88) A \\
\hline & 400 & 8 & $8(100)$ A & $8(100)$ A \\
\hline & 800 & 8 & $8(100)$ A & $8(100) \mathrm{A}$ \\
\hline & 4,000 & 8 & $8(100)$ A & $8(100) \mathrm{A}$ \\
\hline & 8,000 & 8 & $8(100)$ A & $8(100)$ A \\
\hline & 40,000 & 8 & $8(100) \mathrm{A}$ & $8(100)$ A \\
\hline & Total & 48 & $46(96)$ A & 47 (98) A \\
\hline \multirow[t]{7}{*}{ Preevis $^{b}$} & 32 & 8 & $7(88)$ A & $8(100) \mathrm{A}$ \\
\hline & 64 & 8 & $5(63)$ A & $8(100)$ A \\
\hline & 320 & 8 & $8(100)$ A & $8(100) \mathrm{A}$ \\
\hline & 640 & 8 & $8(100)$ A & $8(100)$ A \\
\hline & 3,200 & 8 & $8(100)$ A & $8(100) \mathrm{A}$ \\
\hline & 6,400 & 8 & $8(100) \mathrm{A}$ & $8(100)$ A \\
\hline & Total & 48 & 44 (92) в & $48(100) \mathrm{A}$ \\
\hline
\end{tabular}

${ }^{a}$ Values within a row with the same letter are not different $(P>$ $0.05)$.

${ }^{b}$ Carcass surface samples were collected from beef carcasses prior to evisceration.

were not different $(P>0.05)$ for the recovery of $E$. coli O157:H7 from the inoculated ground beef enrichments. However, when comparing the total number of carcass enrichments from which E. coli $\mathrm{O} 157: \mathrm{H} 7$ was recovered, PIMS was better $(P<0.05)$. While the two methods recovered E. coli $\mathrm{O} 157: \mathrm{H} 7$ from every sample when the inoculum was $320 \mathrm{CFU}$ of E. coli $\mathrm{O} 157$ per $\mathrm{ml}$ or greater, the P-IMS method had a better recovery rate for those enrichments containing lower concentrations of $E$. coli $\mathrm{O} 157: \mathrm{H} 7$.

Comparison of C-IMS and P-IMS for the recovery of $E$. coli 0157:H7 from spiked ground beef. Ground beef samples were inoculated with E. coli $\mathrm{O} 157: \mathrm{H} 7$ at concentrations of 1 to $2 \mathrm{CFU} / 65 \mathrm{~g}$. The inoculated ground beef was then enriched under different conditions, as described in "Materials and Methods." Following enrichment, the recovery efficiency of C-IMS and P-IMS from these ground beef samples was not different $(P>0.05)$, with $E$. coli O157:H7 being recovered from 76 and $77 \%$ of the samples for P-IMS and C-IMS, respectively (data not shown).

These experiments demonstrated that the benefit of the use of P-IMS for the recovery of E. coli $\mathrm{O} 157: \mathrm{H} 7$ in ground beef is the increased speed of sample processing without compromising the sensitivity of detection for $E$. coli $\mathrm{O} 157: \mathrm{H} 7$. In contrast, the isolation of $E$. coli $\mathrm{O} 157: \mathrm{H} 7$ is often hindered in other types of samples, such as carcass or hide sponge samples, by the overgrowth of background microflora that also are able to grow on the selective and differential media used to identify the target pathogen. Efforts have been made to reduce the background growth by diluting starting samples (thus further reducing the volume of sample processed), adding nonspecific binding blockers with beads of different sizes, and increasing the stringency of the washing conditions $(6,13)$, but the background flora continues to make detection of target pathogens problematic.

Comparison of the performance of C-IMS and PIMS for the recovery of $E$. coli $0157: \mathrm{H} 7$ from uninoculated bovine-related samples. To evaluate the C-IMS and P-IMS methods for $E$. coli $\mathrm{O} 157: \mathrm{H} 7$ recovery under more rigorous conditions, the two methods were compared with highly contaminated samples. These samples included cattle feces and hides collected at a feedlot and carcasses obtained at commercial slaughter plants. For all three sample types, P-IMS outperformed $(P<0.05) \mathrm{C}$-IMS in detection of $E$. coli $\mathrm{O} 157: \mathrm{H} 7$ (Table 2). The C-IMS procedure resulted in the isolation of E. coli $\mathrm{O} 157: \mathrm{H} 7$ from 8, 57, and $5 \%$ of carcass, hide, and fecal samples, respectively. In contrast, use of the P-IMS procedure from the same samples resulted in the isolation of E. coli $\mathrm{O} 157: \mathrm{H} 7$ from 12, 82, and $25 \%$ of the carcass, hide, and fecal samples, respectively. While P-IMS missed $1 \%$ of carcass samples that were identified as E. coli O157:H7 positive by C-IMS, it identified an additional 5, 25, and $20 \%$ of carcass, hide, and fecal samples, respectively, as positive for E. coli O157:H7 that were missed by the C-IMS procedure. When all three types of samples were combined, the P-IMS procedure significantly outperformed the C-IMS, as it allowed the detection of $20 \%$ of the positive samples, while C-IMS detected only $12 \%$ of the positive samples. One reason for the increased detection with P-IMS is that carryover of background microflora is lower, making it easier to identify the target pathogen on agar plates (Fig. 1).

A common problem encountered in isolating E. coli O157:H7 from cattle fecal samples by IMS is poor and inconsistent bead recovery (13). Although the fecal micro-

TABLE 2. Recovery of E. coli O157:H7 from uninoculated samples

\begin{tabular}{|c|c|c|c|c|c|c|c|}
\hline \multirow[b]{2}{*}{ Sample type } & \multirow[b]{2}{*}{$\begin{array}{c}\text { No. of } \\
\text { samples }\end{array}$} & \multicolumn{6}{|c|}{ No. $(\%)$ of $E$. coli $\mathrm{O} 157: \mathrm{H} 7$ recovered $^{a}$} \\
\hline & & C-IMS ${ }^{+}$ & P-IMS ${ }^{+}$ & $\begin{array}{c}\text { C-IMS } \\
\text { and } \\
\text { P-IMS }{ }^{+}\end{array}$ & $\begin{array}{c}\text { C-IMS } \\
\text { and } \\
\text { P-IMS }^{-}\end{array}$ & $\begin{array}{c}\text { C-IMS } \\
\text { and } \\
\mathrm{P}^{-} \mathrm{IMS}^{+}\end{array}$ & $\begin{array}{c}\text { C-IMS } \\
\text { and } \\
\text { P-IMS }^{-}\end{array}$ \\
\hline Carcass sponge & 816 & $62(8)$ B & 97 (12) A & 54 & 8 & 43 & 711 \\
\hline Hide sponge & 96 & $55(57) \mathrm{B}$ & $79(82) \mathrm{A}$ & 55 & 0 & 24 & 17 \\
\hline Fecal & 96 & $5(5) \mathrm{B}$ & $24(25) \mathrm{A}$ & 5 & 0 & 19 & 72 \\
\hline Total & 1,008 & $122(12) \mathrm{B}$ & $200(20) \mathrm{A}$ & 114 & 8 & 86 & 800 \\
\hline
\end{tabular}

\footnotetext{
${ }^{a}$ Values within a row with the same letter are not different $(P>0.05)$.
} 
C-IMS

P-IMS

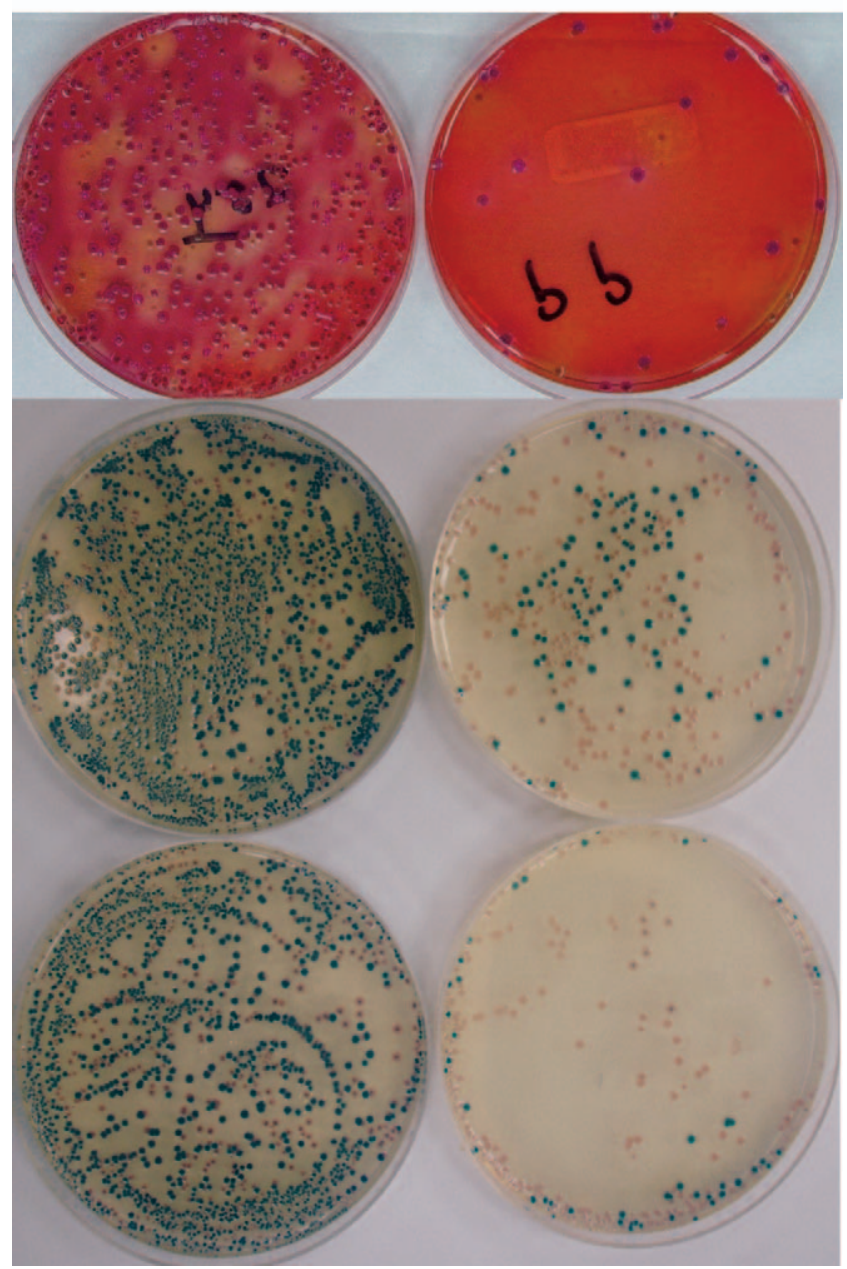

FIGURE 1. Comparison of background growth of preevisceration carcass samples on sorbitol MacConkey agar supplemented with cefixime $(0.05 \mathrm{mg} /$ liter $)$ and potassium tellurite $(2.5 \mathrm{mg} / \mathrm{liter})$ and CHROMagar supplemented with tellurite $(1 \mathrm{mg} / \mathrm{liter})$ and novobiocin (5 mg/liter) plates by IMS with the PickPen and conventional IMS procedures.

flora is expected to be very complex, the overgrowth of background flora on the plates for beads captured from enriched fecal samples, by either C-IMS or P-IMS, was rarely observed. This was most likely due to the inefficiency of bead recovery from fecal samples. The bead recovery efficiency was slightly improved by the P-IMS procedure, although it was still lower than that found with other types of samples. This increased bead recovery is most likely responsible for the observed improvement (5 versus 25\%) in $E$. coli $\mathrm{O} 157: \mathrm{H} 7$ detection from fecal samples. With larger beads (Aureon Biosystems, Vienna, Austria), we observed further improvement in bead recovery with the PIMS procedure. The Aureon beads are $7.0 \mu \mathrm{m}$ in diameter, while the Dynal beads have a diameter of $2.8 \mu \mathrm{m}$. No significant increase of $E$. coli $\mathrm{O} 157: \mathrm{H} 7$ detection was observed with larger beads (data not shown). This may be because of the differences in antibodies (e.g., affinity, concentrations) that are used by each manufacturer.

Overall, P-IMS results in lower carryover of background microflora to the plating media than does C-IMS, which facilitates the identification of potentially positive colonies (Fig. 1). Data presented here demonstrate that $E$. coli $\mathrm{O} 157: \mathrm{H} 7$ detection efficiency was greatly improved by the P-IMS procedure, especially when the target samples were being isolated from enrichments containing a high background of microflora.

Evaluation of sequential P-IMS of multiple pathogens from the same sample. The PickPen device removes the immunobeads from the sample, instead of removing the sample from the tube containing the beads; thus, it becomes a simple matter to use the same sample to detect the presence of multiple pathogens. Additional immunobeads, specific for the next pathogen of interest, are added to the sample wells following the initial IMS, and the new beads are then incubated, washed, and recovered.

To determine if IMS for E. coli O157:H7 interfered with the subsequent ability to isolate Salmonella by IMS, or vice versa, enrichment samples (1-ml volume) were subjected to sequential IMS by the addition of $20 \mu l$ of either E. coli 0157:H7- or Salmonella-specific magnetic beads. The beads were extracted from enrichment samples with the PickPen device as described in "Materials and Methods." Next, $20 \mu \mathrm{l}$ of Salmonella or E. coli O157:H7 beads (those not previously added) was added to the enrichments and again removed with the PickPen as described. Regardless of which pathogen-specific beads were used first, the same $(P>0.05)$ amount of E. coli O157:H7 or Salmonella was detected (Table 3). Thus, both E. coli O157:H7 and Salmonella can be isolated from the same enrichment aliquot, when both are present, and the results will be independent of the order of bead addition.

A successful food safety program requires rapid and efficient detection of foodborne pathogens. For the meat industry, E. coli O157:H7 is a primary concern. Despite the rapid advancement of modern technologies, such as real-

TABLE 3. Sequential IMS for E. coli O157:H7 and Salmonella

No. $(\%)$ of samples positive for $^{a}$ :

Method

No. of samples

E. coli $\mathrm{O} 157: \mathrm{H} 7$

Salmonella

E. coli $\mathrm{O} 157: \mathrm{H} 7$ beads first and then

Salmonella beads

$62(94)$ A

$47(71) \mathrm{A}$

Salmonella beads first and then E. coli

O157:H7 beads

66

$63(95)$ A

$45(68)$ A

\footnotetext{
${ }^{a}$ Values within a column with the same letter are not different $(P>0.05)$
} 
time PCR, in the field of pathogen detection, IMS is still an indispensable tool for this task. IMS is often integrated into newly developed pathogen detection protocols and is widely applied to concentrate specific pathogens from a large volume of samples. The captured pathogens can then be detected by a variety of methodologies, from real-time PCR to simple plating on selective media. In addition, the potential that sequential IMS has for the enrichment of samples for the isolation of multiple pathogens further extends the utility and flexibility of this tool. The PickPen magnetic particle transfer device, with its 96-well microplate compatibility design, can be easily adapted for high-throughput pathogen detection and future automation. In our routine surveys of E. coli O157:H7 and Salmonella prevalence in various sample types in which high-throughput IMS is essential, we have consistently observed great reductions in sample processing times when PickPen devices were used. Use of the PickPen in IMS provides these advantages: (i) high-throughput IMS, (ii) consistent recovery of immunobeads, and (iii) lower carryover of background microflora.

\section{ACKNOWLEDGMENTS}

The authors thank Julie Dyer, Bruce Jasch, Frank Reno, and Greg Smith for technical support and Carol Grummert for secretarial assistance.

\section{REFERENCES}

1. Anonymous. 2005. An outbreak of Escherichia coli O157:H7 associated with a children's water spray park and identified by two rounds of pulsed-field gel electrophoresis testing. Can. Commun. Dis. Rep. 31:133-140.

2. Arthur, T. M., J. M. Bosilevac, M. P. Kent, D. Jaroni, X. Nou, S. D. Shackelford, T. L. Wheeler, B. Pauling, D. M. Allen, and M. Koohmaraie. 2004. Escherichia coli $\mathrm{O} 157$ prevalence and enumeration of aerobic bacteria, Enterobacteriaceae, and Escherichia coli $\mathrm{O} 157$ at various steps in commercial beef processing plants. J. Food Prot. 67:658-665.

3. Arthur, T. M., J. M. Bosilevac, X. Nou, and M. Koohmaraie. 2005. Evaluation of culture- and PCR-based detection methods for Escherichia coli $\mathrm{O} 157: \mathrm{H} 7$ in inoculated ground beef. J. Food Prot. 68: 1566-1574.
4. Barkocy-Gallagher, G. A., T. M. Arthur, M. Rivera-Betancourt, X Nou, S. D. Shackelford, T. L. Wheeler, and M. Koohmaraie. 2003. Seasonal prevalence of Shiga toxin-producing Escherichia coli, including O157:H7 and non-O157 serotypes, and Salmonella in commercial beef processing plants. J. Food Prot. 66:1978-1986.

5. Barkocy-Gallagher, G. A., E. D. Berry, M. Rivera-Betancourt, T. M. Arthur, X. Nou, and M. Koohmaraie. 2002. Development of methods for the recovery of Escherichia coli O157:H7 and Salmonella from beef carcass sponge samples and bovine fecal and hide samples. $J$. Food Prot. 65:1527-1534.

6. Barkocy-Gallagher, G. A., K. K. Edwards, X. Nou, J. M. Bosilevac, T. M. Arthur, S. D. Shackelford, and M. Koohmaraie. 2005. Methods for recovering Escherichia coli O157:H7 from cattle fecal, hide, and carcass samples: sensitivity and improvements. J. Food Prot. 68: 2264-2268.

7. Chapman, P. A. 2000. Methods available for the detection of Escherichia coli $\mathrm{O} 157$ in clinical, food and environmental samples. World J. Microbiol. Biotechnol. 16:733-740.

8. Chapman, P. A., D. J. Wright, and C. A. Siddons. 1994. A comparison of immunomagnetic separation and direct culture for the isolation of verocytotoxin-producing Escherichia coli $\mathrm{O} 157$ from bovine faeces. J. Med. Microbiol. 40:424-427.

9. Durso, L. M., K. Reynolds, N. Bauer, and J. E. Keen. 2005. Shigatoxigenic Escherichia coli O157:H7 infections among livestock exhibitors and visitors at a Texas county fair. Vector Borne Zoonotic Dis. 5:193-201.

10. Fegan, N., G. Higgs, P. Vanderlinde, and P. Desmarchelier. 2004. Enumeration of Escherichia coli $\mathrm{O} 157$ in cattle faeces using most probable number technique and automated immunomagnetic separation. Lett. Appl. Microbiol. 38:56-59.

11. Ferguson, D. D., J. Scheftel, A. Cronquist, K. Smith, A. Woo-Ming, E. Anderson, J. Knutsen, A. K. De, and K. Gershman. 2005. Temporally distinct Escherichia coli $\mathrm{O} 157$ outbreaks associated with alfalfa sprouts linked to a common seed source-Colorado and Minnesota, 2003. Epidemiol. Infect. 133:439-447.

12. Naugle, A. L., K. G. Holt, P. Levine, and R. Eckel. 2005. Food Safety and Inspection Service regulatory testing program for Escherichia coli $\mathrm{O} 157: \mathrm{H7}$ in raw ground beef. J. Food Prot. 68:462-468.

13. Parham, N., J. Spencer, D. Taylor, H. Ternent, G. Innocent, D. Mellor, M. Roberts, and A. Williams. 2003. An adapted ImmunoMagnetic cell separation method for use in quantification of Escherichia coli $\mathrm{O} 157: \mathrm{H} 7$ from bovine faeces. J. Microbiol. Methods 53: $1-9$.

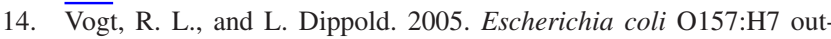
break associated with consumption of ground beef, June-July 2002 Public Health Rep. 120:174-178. 